\title{
Automated Image Analysis of Live/Dead Staining of the Fungus Aureobasidium pullulans on Microscope Slides and Leaf Surfaces
}

BioTechniques 29:874-882 (October 2000)

\author{
Christopher D. Nelson, \\ Russell N. Spear and \\ John H. Andrews \\ University of Wisconsin, \\ Madison, WI, USA
}

\section{INTRODUCTION}

The assessment of cell viability status is of interest in both environmental and medical microbiology $(4,18,22)$. Historically, both indirect and direct methods are used in the determination. In the indirect technique, which equates viability with growth, the viable component of a population is enumerated as colony-forming units (cfu) based on plate counts, and the nonviable component is inferred by subtraction of the cfu from a total microscopic count (13). The accuracy of this method is questionable with the relatively recent recognition of cells that are apparently viable but are unable to be cultured $(1,10)$.

Direct methods entail staining one cell component either viable (live) or nonviable (dead) and obtaining the other component by subtraction from a total cell count $(10,11)$. Live indicators are of two types. First, tetrazolium salts, which are redox indicators, may be used to obtain either colored formazan deposits (2) or fluorescent derivatives such as 5cyano-2,3-ditolyl tetrazolium chloride (CTC) (16). Second, more commonly used indicators are enzyme substrates that are colorless until cleaved by the target enzymes, which then form fluorescent byproducts. Examples include fluorescein diacetate (FDA) and carboxyfluorescein diacetate (CFDA) $(3,6)$. The principle for dead cell staining is based on uptake of dyes (e.g., trypan blue) or fluorochromes (e.g., propidium iodide) by cells with compromised membranes (7). Such compounds are excluded from healthy cells.
The most rigorous direct protocols involve combination staining for both live and dead cells, rather than inferring one subpopulation by assessing the other. Operationally, however, dual estimations are difficult to make. For example, in the case of the tetrazoliums, the redox reaction is relatively slow and needs prolonged incubation to obtain a visible product. During this time, cell death can occur, which influences the estimation of viable cells. Additionally, there may be a species-specific effect from differences in redox potentials of the cells (2). Fluorescent enzyme substrates such as FDA and CFDA are poorly retained in cells after the formation of the fluorescent product; they are sensitive to environmental conditions such as $\mathrm{pH}$ or can photobleach rapidly upon fluorescence excitation (5). While several commercial dual staining kits exist, typically they have been tested on very few organisms such as bacteria or mammalian cells. They also possess limited color selection for those applications that require spectra other than green or red, or may stain only certain organelles (12) of the target cells, making enumeration difficult.

We report here a protocol based on CellTracker Blue ${ }^{\mathrm{TM}}$, a glutathione $S$-transferase-specific live stain, com bined with DEAD Red ${ }^{\mathrm{TM}}$, a dead-cellpermeant stain (both from Molecular Probes, Eugene, OR, USA). This procedure has been applied to viability assessments of blastospores (also to the swollen cell morphotype) of the phylloplane inhabiting yeast-like fungus $A u$ reobasidium pullulans (de Bary) Ar- 
Table 1. Boolean Logic Operators for the Three-Color (Red, Green and Blue) Scheme Used in Master.mac and Their Interpretation by Staining Reaction as Used in Live/Dead Staining Protocol ${ }^{\mathrm{a}}$

\begin{tabular}{|c|c|c|c|}
\hline Fluorochrome(s) & $\begin{array}{l}\text { Boolean } \\
\text { Operators }\end{array}$ & $\begin{array}{c}\text { Resultant Cell } \\
\text { Colorb }\end{array}$ & $\begin{array}{c}\text { Cell } \\
\text { Classification }\end{array}$ \\
\hline DEAD Red & {$[R$ and not $(G$ or $B)]$} & red & Dead wild-type \\
\hline CellTracker Blue & [B and not (R or $\mathrm{G})]$ & blue & Live wild-type \\
\hline Dead Red and GFP & $[R$ and $G$ and (not $B)]$ & yellow & Dead GFP \\
\hline CellTracker Blue and GFP & [B and $G$ and (not R)] & cyan & Live GFP \\
\hline $\begin{array}{l}\text { DEAD Red and } \\
\text { CellTracker Blue }\end{array}$ & [R and $B$ and (not $G$ )] & magenta & $\begin{array}{l}\text { Compromised } \\
\text { wild-type }\end{array}$ \\
\hline $\begin{array}{l}\text { DEAD Red and } \\
\text { CellTracker Blue and GFP }\end{array}$ & (R and $\mathrm{G}$ and $\mathrm{B}$ ) & white & Compromised GFP \\
\hline $\begin{array}{l}\text { aSee also Figure } 4 . \\
\text { bFrom combined image planes. }\end{array}$ & & & \\
\hline
\end{tabular}

naud (Deuteromycetes; Moniliales). Further, to demonstrate its general utility, we have sucessfully applied the procedure to live and dead cells of the yeast Cryptococcus laurentii (Kuff.) Skinner. A third marker, such as green fluorescent protein (GFP), can also be imaged. Cells stained by this method are relatively resistant to photobleaching and may be preserved for image analysis at a later time. This is an added benefit for experiments in which many samples must be processed concurrently. We also describe the computer programs that assist in digital image acquisition and quantitative image analysis. Assessments can be made of cell populations on microscope slides, or cells can be stained in situ on leaf surfaces.

\section{MATERIALS AND METHODS}

Cultures of $A$. pullulans (ATCC no. 90393) and a TEFE GFP transformant of this cell line (20) were maintained on yeast peptone glucose (YPG) medium slants at room temperature or $4^{\circ} \mathrm{C}$. Details regarding the GFP-transformed line appear elsewhere (20), but, in brief, the TEFE GFP construct consists of about 800 bp of the $5^{\prime}$-untranslated region of the translation elongation factor (TEF) gene from A. pullulans fused to an engineered form of GFP (EGFP), followed by a 200-bp terminator region of the Aspergillus awamori glucoamylase gene. The EGFP is a red-shifted variant (Clontech Laboratories, Palo Alto, CA, USA) with mutations in the chromophore that shift the maximum excitation peak from $395 \mathrm{~nm}$ to approximately $490 \mathrm{~nm}$. Working cultures of both lines were grown in liquid YPG medium with constant shaking at 125 $\mathrm{rpm}$ at room temperature. Predominately live cell populations $(>80 \%)$ were from overnight cultures, while predominately dead cells (>99\%) were obtained by nutrient depletion following 12-14 days of shake culture. Cell death was presumed by the absence of growth on YPG plates following a three-day incubation at room temperature. Concentrations of live and dead cells were determined by hemacytometer counts and were adjusted to $1 \times 10^{7}$ cells $/ \mathrm{mL}$ with liquid YPG. Standardized mixtures of live and dead cells were prepared by combining the populations in live:dead cell ratios of $1: 0,3: 1,1: 1,1: 3$ and $0: 1$.

All subsequent procedures were conducted in subdued light to prevent photobleaching of the fluorochromes, which should be treated as potential mutagens with appropriate safety precautions being followed. Two microliters of a stock solution of CellTracker Blue CMHC (4-chloromethyl-7-hydroxycoumarin; $5 \mathrm{mg}$ dissolved in 2 $\mathrm{mL}$ DMSO and stored at $-20^{\circ} \mathrm{C}$ ) were added to $500 \mu \mathrm{L}$ cell suspension and incubated for $1 \mathrm{~h}$ at room temperature in the dark. The cells were washed twice by centrifugation and resuspension in YPG and incubated in YPG for another hour to allow full conversion of the CellTracker probe. To prevent high background fluorescence, excess CellTracker was removed by two washes with YPG. Then, cells were washed as above with two changes of distilled water. DEAD Red (ethidium homodimer2) was prepared by adding $2 \mu \mathrm{L}$ supplied stock solution to $1 \mathrm{~mL}$ distilled water. Fifty microliters of the stain were added to $50 \mu \mathrm{L}$ cells suspended in distilled water and incubated for $30 \mathrm{~min}$ in the dark. Cell suspensions were washed twice as above with distilled water before fixation with $2 \%$ formaldehyde (prepared from para-formaldehyde) in $10 \mathrm{mM}$ PBS, pH 7.2, for 15 $30 \mathrm{~min}$. Cells were washed twice with distilled water and resuspended in 250 $\mu \mathrm{L}$ distilled water. Cell suspensions (5$\mu \mathrm{L}$ drops) were applied to polylysinecoated, 12-well Teflon ${ }^{\circledR}$ printed microscope slides (Electron Microscopy Sciences, Fort Washington, PA, USA) and air dried at room temperature. Slides were stored in slide boxes, unmounted at $4^{\circ} \mathrm{C}$ for several days before imaging. Immediately before imaging, the preparations were mounted in antifade VECTASHIELD ${ }^{\circledR}$ (Vector Laboratories, Burlingame, CA, USA) and cover slips applied.

We obtained images of the fluorescent cells using an Olympus BX-60 fluorescence microscope (Olympus America, Lake Success, NY, USA), equipped with a 100-W Hg arc lamp, a Chroma 83000 filter set (Chroma Tech- 
nology, Brattleboro, VT, USA) and an Optronics DEI 470 cooled CCD camera (Optronics Engineering, Goleta, CA, USA). Three images were acquired of the same field in the following order. First, a red image (dead cells) was acquired by using a standard propidium iodide excitation filter (Chroma 83570, $570 \mathrm{~nm}$ ). Next, a green signal image for GFP (or similar fluorochrome) was taken with a standard FITC excitation (Chroma 83490, $490 \mathrm{~nm}$ ) filter. Finally, a blue image (live cells) was acquired with a narrow band UV excitation (Chroma 3013, $365 \pm 10 \mathrm{~nm}$ ) filter. An auxiliary barrier (Chroma HQ $46320 \mathrm{M}$, $463 \mathrm{~nm} \pm 20 \mathrm{~nm}$ ) filter was also used when GFP was used as a third signal.

A macro program acquire.mac (Figure 1) was written to obtain the images and to run under the image analysis

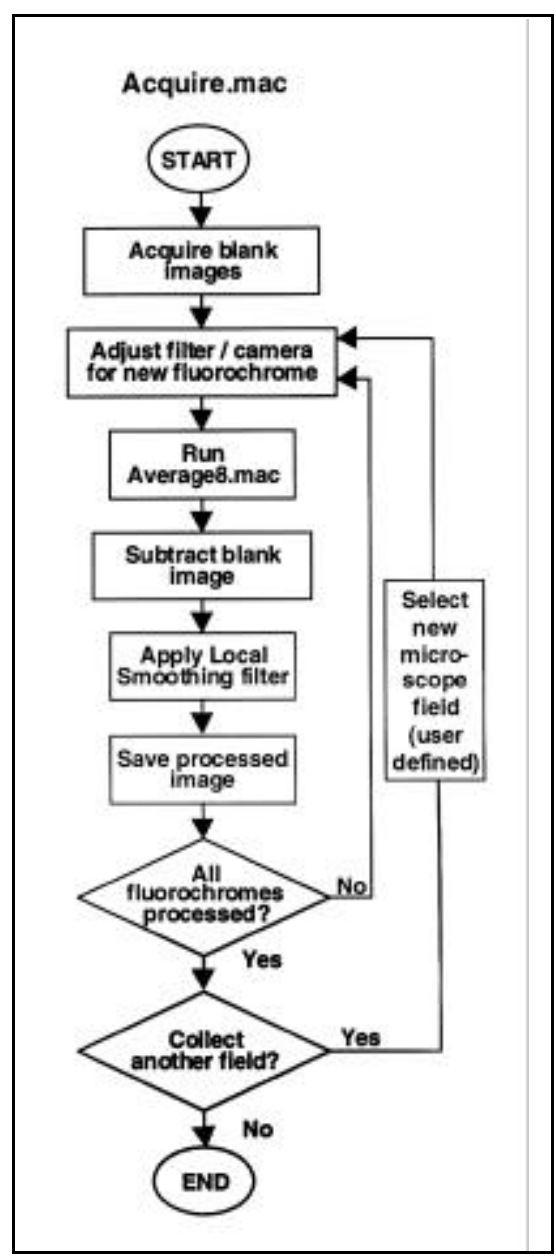

Figure 1. Acquire.mac. Processing flowchart of the image acquisition macro that prompts the user for microscope filter and exposure adjustments and automatically saves images before processing. software Optimas $^{\mathrm{TM}} 6.2$ (Media Cybernetics, Bothell, WA, USA) on a Pentium $^{\mathrm{TM}}$ (Intel, Santa Clara, CA, USA) 166-MHz personal computer equipped with a Flash Point 128 (Integral Technologies, Indianapolis, IN, USA) frame grabber board. The acquire macro prompted the user to manually set the proper filter for each color channel (red, R; green, G; blue, B) and allowed focus and exposure adjustments. After a focused, correctly exposed image was

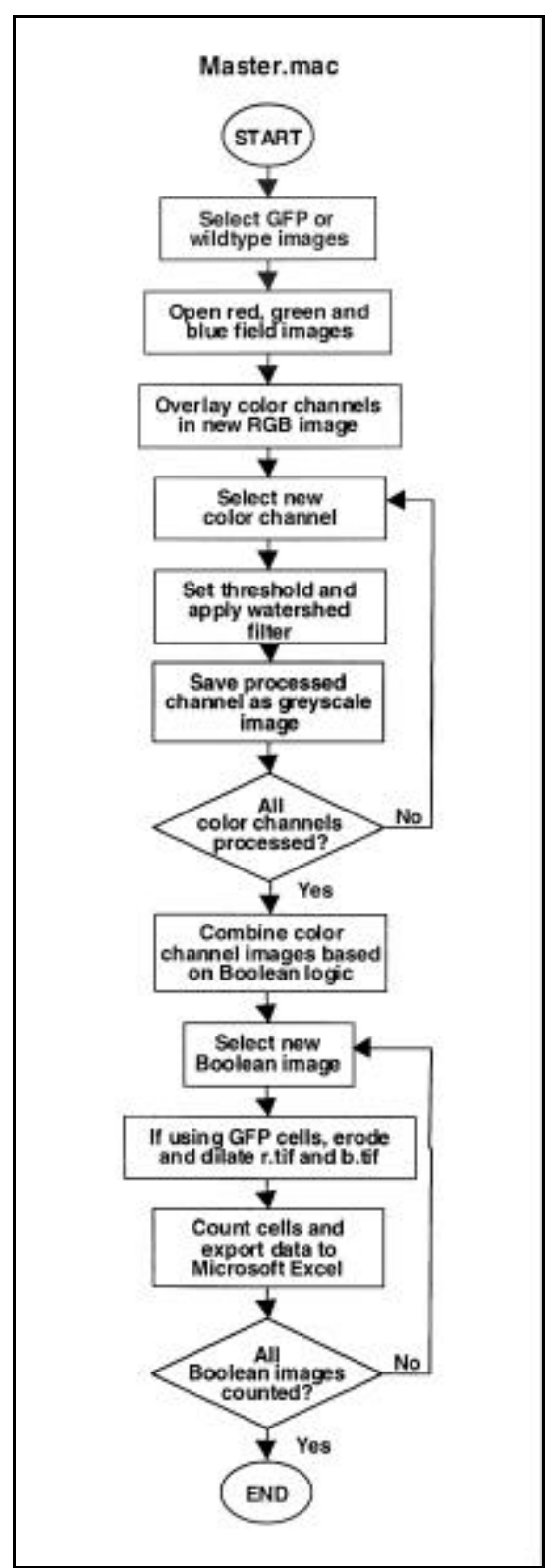

Figure 2. Master.mac. Processing flowchart of the live/dead image analysis program macro that enumerates and exports live, dead and compromised cell counts to Excel spreadsheets. obtained, the program averaged eight images of the field to reduce temporal noise and subtracted a blank image (illumination shutter closed) to reduce spatial noise. Finally, a localized background-smoothing filter was applied to delineate cells that were apposed. These steps were essential for successful global, automatic threshold determination later in the image analysis (21). Then, the macro saved three 8-bit color TIFF files (red $x$.tif, green $x$.tif and bluex.tif, where $x$ is the corresponding set number) to a user-designated directory for further image analysis.

Enumeration of the labeled cells was performed with the macro program master.mac (Figure 2) also running under Optimas 6.2. Processing began when the user selected the appropriate color channels from the three images (e.g., taking the red channel from the DEAD Red image) and combined them to make a new RGB image. This was designated overlay.tif and was saved to a new user-defined directory along with all subsequent intermediate images.

To separate the features of the im ages from the background, each color channel underwent a thresholding procedure. The macro selected an initial threshold value based on the Otsu minimal variance method of threshold selection (14) for the red and green values and Search for Minimum for the blue value. If this threshold was unacceptable because of the exclusion of cells or inclusion of background noise, the user made the appropriate adjustments to lower or raise the threshold, respectively. When a threshold had been set, a watershed separation filter $(17,21)$ was applied to separate clusters of cells and to produce a binary image of each channel. A series of Boolean logical operators (Table 1) was applied to the binary color channels to determine regions of overlapping or exclusive staining (17). The grayscale Boolean images were counted by using the Optimas "count areas" command and the data were exported via a DDE link to a Microsoft ${ }^{\circledR}$ Excel $97^{\circledR}$ spreadsheet for interpretation and presentation. Three experiments were conducted with wild-type cells and two with GFP-transformed cells.

For leaf surface studies, a mixture of cells from overnight cultures of wildtype and EGFP-transformed A. pullu- 
lans was placed on detached tobacco (Nicotiana tabacumL. cv Havana) leaf segments (about $4 \mathrm{~cm}^{2}$ ) for $2 \mathrm{~h}$. Excess moisture was removed with a pipet, and the segments were inverted onto drops of stain on plastic film. Leaf segments then were washed by dipping them into water in petri dishes, drained and mounted in VECTASHIELD before examination. Images were acquired by using the filter sets described above; however, only a single image of each fluorochrome was taken, and a blank image subtraction was not performed. Image analysis was done the same as with the cells on microscope slides. This experiment was conducted twice.

\section{RESULTS AND DISCUSSION}

Typical live versus dead cell staining results for wild-type and GFP-transformed A. pullulans are shown in Figure 3 for the GFP cell line. Dead cells always occurred in the standardized live cell population, but no live cells were seen in the dead cell population. It was therefore necessary to correct the observed results to account for the dead cells observed in what was supposed to be an exclusively live cell population for experimental purposes. We calcu- lated this adjustment by using Minitab v12.0 (Minitab, State College, PA, USA) from the linear regression of the actual proportions (dead/total) versus the expected proportions (dead/total) for the five ratios of cells. As there were negligible live cells in the dead population, the regression line was forced to go through the origin $(y=0, x=0)$, where $y$ is the observed proportion of dead cells. The estimated slope is the proportion of "cells that should be live" that are observed to be live. The reciprocal of this slope (what we call the $\alpha$-factor) is used as a multiplier to correct observed data values. We note that this method assumes that the proportion of dead cells observed in the live sample is constant across the ratios.

Cells showing both blue and red staining were interpreted as compromised and counted as dead. That a cell can register both live and dead reactions is explicable by the relatively long half-life (about $60 \mathrm{~min}$ ) (19) of glutathione $S$-transferase, implying that recently dead cells will continue to stain blue. This is further supported by the observation that suspensions of live cells contained compromised cells, while suspensions of the nutrient-depleted dead cells did not.

To determine whether our protocol

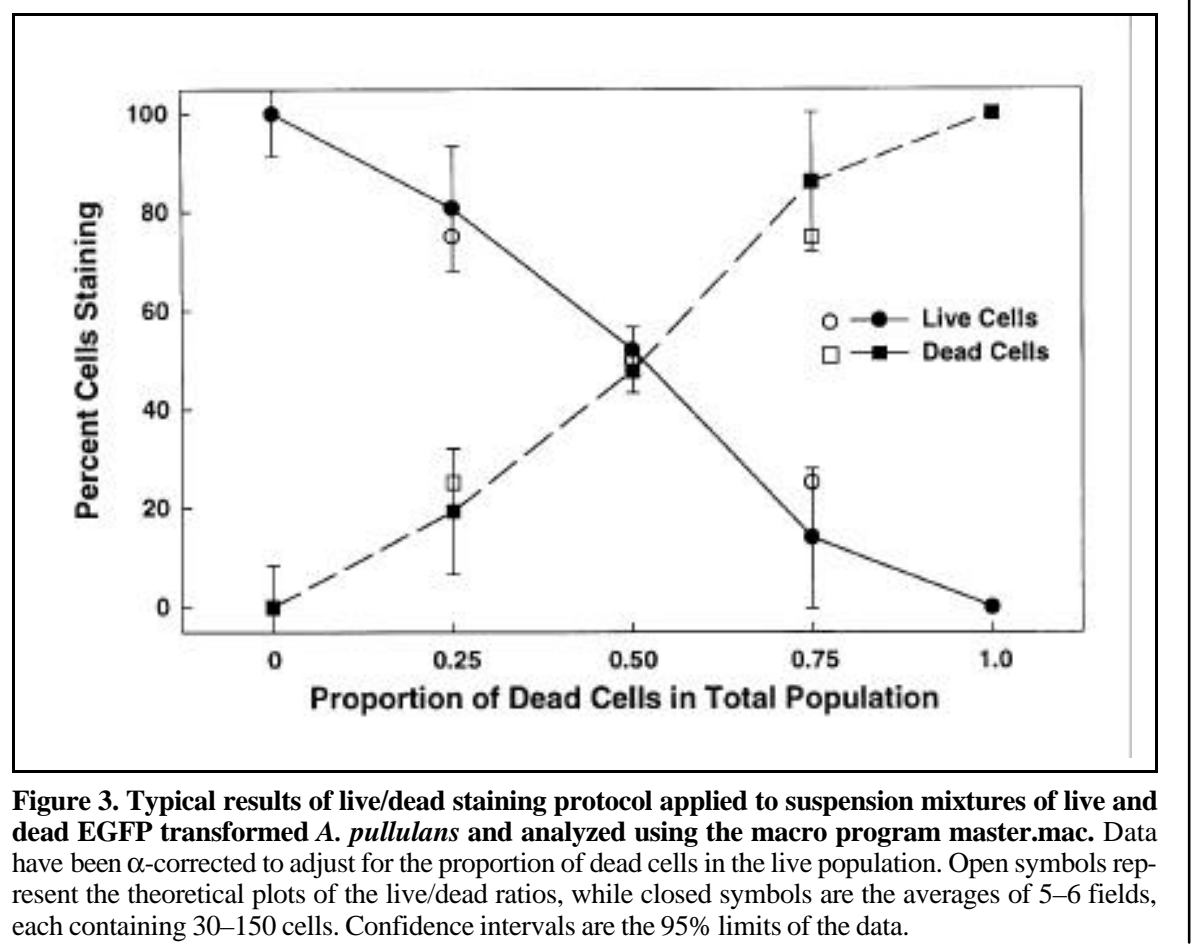




\section{Research Report}

was applicable to other fungi, live and dead cell suspensions of Cryptococcus laurentii (ATCC no. 32063) were stained and produced results similar to A. pullulans (data not shown). We also
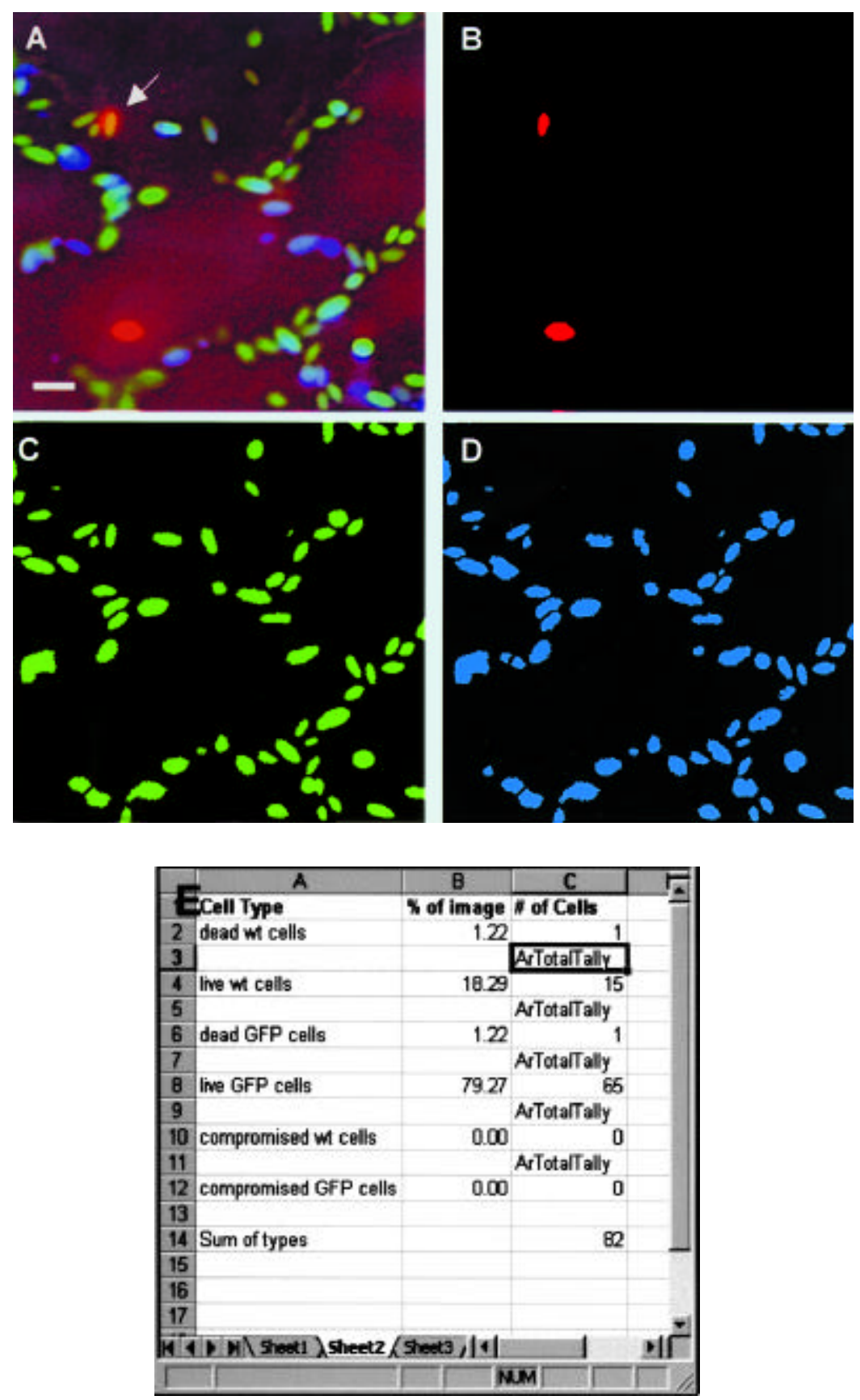

Figure 4. Results of live/dead staining, GFP expression and processed image data. (A) Live/dead staining reaction and GFP expression of a mixture of wild-type and EGFP-transformed cells of A. pullulans applied to a tobacco leaf [the image has been digitally deconvolved to enhance focus using Microtome $^{\mathrm{TM}}$ (VayTek, Fairfield, IA, USA) deconvolution software for illustrative purposes only]; $\rightarrow$ indicates a dead cell also expressing GFP. (B-D) Binary images of the respective red, green and blue components of the processed image, pseudo-colored for clarity. (E) Excel output of the image shown in (A) (compare interpretation with Boolean operators logic, Table 1). Scale bar equals $10 \mu \mathrm{m}$. and $\operatorname{dead}$ A. pullulans cells, which produced results similar to those produced using CellTracker and DEAD Red.

Figure 4 shows the original RGB im age of live and dead cells (Figure 4A) in a mixture of wild-type and EGFP-expressing cells on a leaf surface, together with the binary images for the red, green and blue signals, respectively (falsely colored for illustration) (Figure 4, B, C and D), and the Excel ${ }^{\circledR}$ output of the master.mac (Figure 4E). Although red autofluorescence of chlorophyll is seen in the unprocessed image of the leaf (Figure 4A), the red signal of the dead-staining cells is detected by applying a threshold to discriminate cells from background. Image averaging and background subtraction were not applied to images of cells on leaves because elimination of background was not possible and image averaging required additional time during which photobleaching of the chlorophyll occurs. Work is in progress to control photobleaching, which would allow image averaging; this in turn would permit collection of a less noisy signal (especially in the blue channel, which is the least sensitive for CCD cameras) (15).

GFP has an excitation spectrum that must be compensated for to obtain accurate results. The UV excitation used for CellTracker Blue will also excite GFP, causing EGFP-expressing cells to be seen in the blue and green channels. This is evident visually in the blue detection channel as either cyan-colored cells, in the case of cells having both CellTracker and EGFP signals, or as green in those cells that have GFP and DEAD Red signals (as red does not overlap into blue). This effect was quite pronounced in our EGFP-transformed cell line, and the problem had to be corrected before image processing to prevent green- and red-expressing cells (categorized as GFP and Dead) from being interpreted as expressing green, red and blue (GFP and compromised). This was corrected in two ways. First, we changed the excitation filter from the standard $360 \mathrm{~nm} \pm 40 \mathrm{~nm}$ to $365 \mathrm{~nm}$ $\pm 10 \mathrm{~nm}$ (Chroma Technology) and inserted a neutral density filter of $3.0 \mathrm{OD}$ units (Olympus America) in the light path, allowing less excitation of the unwanted GFP signal. Second, an additional barrier filter HQ $463 \pm 20 \mathrm{~nm}$ 


\section{Research Report}

(Chroma Technology) was used to further reject non-blue light spilling over into the blue image. This signal overlap is not a problem with fluorescein, which does not have an excitation spectrum extending into the shorter wavelengths.

The macro programs written to process the fluorescent images are accurate as validated by manual counts of live and dead cells. However, they have the following potential to generate errors. Chromatic aberration of the microscope optics, inadvertent movement of the microscope stage or differences in cellular localization of probes can lead to a mis-registration of fields when the component color channels are overlaid. The resulting non-overlapping areas can be large enough to be counted as individual cells, thereby causing erroneous counts. We have encountered this problem with the cellular distribution of EGFP in transformed cells being more centralized than the live/dead probes, leaving "halos" of red or blue around a green center. Addition of an extra binary erosion of the blue and red images before enumeration of the live and dead cells in the GFP images eliminated this artifact.

As applying threshold values to the images is a subjective step in the processing, it was apparent early in the macro development that some variation among users was a factor when discrim inating dim from bright cells. To minimize this problem, we integrated the Optimas auto-thresholding feature to locate an initial starting threshold. The Otsu minimal variance method (14) was selected for the red and green channels, and the more conservative "Search for Minimum" method from the Optimas program was applied to the blue channel. These adjustments corrected for the relative insensitivity of the cooled CCD camera to blue. Consequently, the user rendered a noisier image, which al- lowed establishment of a consensus threshold level that needed only occasional fine adjustment.

Sometimes the master.mac will cause computer system lock ups when exporting data to Excel 97. This is a conflict between Windows $95 / 98^{\mathrm{TM}}$ and the DDE function and appears to be related to the system running the program. We have written the routine reset.mac to allow recovery of the spreadsheet without data loss and to continue processing images.

The macro programs are available for downloading as live_mac from the Software Library of the BioTechniques Web site (www.biotechniques.com) by anonymous ftp from (plantpath.wisc. edu) or by email from the authors (rzs @ plantpath.wisc.edu) free of charge. The documentation and tutorial contain more information and advice on the im age-processing techniques used by this software. Though these macros are 
written in the ALI language used by Optimas, programmers familiar with the $\mathrm{C}$ language will have little trouble modifying the code to run under image processing packages such as Image Pro Plus $^{\mathrm{TM}}$ (Media Cybernetics).

In summary, this protocol and imaging program macros allow the enumeration of live versus dead cells, plus a third parameter with accuracy unattainable manually because of time constraints or impossible because of photobleaching. Because samples can be fixed after the live/dead staining, specimens can be stored before the examination or application of another probe. Furthermore, extension of this method to the plant surface allows the direct, in situ assessment of the potential viability status of resident populations of native wild-type or introduced EGFP-expressing microorganisms associated with epiphytic landscape features of interest. The method could also be combined with fluorescently labeled in situ hybridization probes $(8,9)$ for the identification of specific microbe species or with fluorescently labeled lectins or antibodies.

\section{ACKNOWLEDGMENTS}

This work was supported by USDA Hatch project no. 142-3995. We thank William A. Russin for reviewing the manuscript and Erik V. Nordheim for advice on the statistics.

\section{REFERENCES}

1.Barer, M.R. and C.R. Harwood. 1999. Bacterial viability and culturability, p. 93-137. In R.K. Poole (Ed.), Advances in Microbial Physiology. Academic Press, San Diego.

2.Barka, T. and P.J. Anderson. 1963. Histochemistry Theory, Practice, and Bibliography. Harper \& Row, New York.

3.Diaper, J.P. and C. Edwards. 1994. The use of fluorogenic esters to detect viable bacteria by flow cytometry. J. Appl. Bacteriol. 77:221228.

4.Diaper, J.P., K. Tither and C. Edwards. 1992. Rapid assessment of bacterial viability by flow cytometry. Appl. Microbiol. Biotechnol. 38:268-272.

5.Haugland, R.P. 1996. Handbook of Fluorescent Probes and Research Chemicals, 6th ed. Molecular Probes Inc., Eugene, OR.

6.Jacobsen, C.N., J. Rasmussen and M. Jakobsen. 1997. Viability staining and flow cytometric detection of Listeria monocytogenes. J. Microbiol. Methods 28:35-43.

7.Jones, K.H. and J.A. Senft. 1985. An im proved method to determine cell viability by simultaneous staining with fluorescein diacetate-propidium iodide. J. Histochem. Cytochem. 33:77-79.

8.Li, S., D. Cullen, M. Hjort, R. Spear and J.H. Andrews. 1996. Development of an oligonucleotide probe for Aureobasidium pullulans based on the small-subunit rRNA gene. Appl. Environ. Microbiol. 62:1514-1518.

9.Li, S., R.N. Spear and J.H. Andrews. 1997. Quantitative fluorescence in situ hybridization of Aureobasidium pullulans on microscope slides and leaf surfaces. Appl. Environ. Microbiol. 63:3261-3267.

10.Lloyd, D. and A.J. Hayes. 1995. Vigour, vitality and viability of microorganisms. FEMS Microbiol. Lett. 133:1-7.

11.McFeters, G.A., F.P. Yu, B.H. Pyle and P.S. Stewart. 1995. Physiological assessment of bacteria using fluorochromes. J. Microbiol. Methods 21:1-13.

12.Millard, P.J., B.L. Roth, H.P. Truong, S.T. Yue and R.P. Haugland. 1997. Development of the FUN-1 family of fluorescent probes for vacuole labeling and viability testing of yeasts. Appl. Environ. Microbiol. 63:28972905.

13.Murray, R.G.E., R.N. Doetsch and C.F. Robinow 1994. Determinative and cytological light microscopy, p. 21-41. In P. Gerhardt,
R.G.E. Murray, W.A. Wood and N.R. Krieg (Eds.), Methods for General and Molecular Bacteriology. American Society for Microbiology. Washington, DC.

14.Otsu, N. 1979. A theshold selection method from gray-level histograms. IEEE Trans. Syst. Man Cybern. SMC 9:62-66.

15.Pawley, J.B. 1994. The sources of noise in three-dimensional microscopical data sets, $\mathrm{p}$. 48-94. In J.K. Stevens, L.R. Mills and J.E. Trogadis (Eds.), Three-Dimensional Confocal Microscopy: Volume Investigation of Biological Specimens. Academic Press, New York.

16.Rodriguez, G.G., D. Phipps, K. Ishiguro and H.F. Ridgway. 1992. Use of a fluorescent redox probe for direct visualization of actively respiring bacteria. Appl. Environ. Microbiol. 58:1801-1808.

17.Russ, J.C. 1999. The Image Processing Handbook, 3rd ed. CRC Press, Boca Raton FL.

18.Taghi, K.R., L.L. Gyürek, P.J. Millard, G.R. Finch and M. Belosevic. 1997. Nucleic acid stains as indicators of Cryptosporidium parvum oocyst viability. Int. J. Parasitol. 27:787-798.

19.Trull, A.K., S.P. Facey, G.W. Rees, D.G.D. Wight, G. Noble-Jamieson, C. Joughin, P.J. Friend and G.J.M. Alexander. 1994. Serum $\alpha$-glutathione s-transferase-a sensitive marker of hepatocellular damage associated with acute liver allograft rejection. Transplantation 58:1345-1351.

20.Vanden Wymelenberg, A.J., D. Cullen, R.N. Spear, B. Schoenike and J.H. Andrews. 1997. Expression of green fluorescent protein in Aureobasidium pullulans and quantification of the fungus on leaf surfaces. BioTechniques 23:686-690.

21.Wilkinson, M.H.F. 1998. Automated and manual segmentation techniques in image analysis of microbes, p. 135-171. In M.H.F. Wilkinson and F. Schut (Eds.), Digital Image Analysis of Microbes: Imaging, Morphometry, Fluorometry and Motility Techniques and Applications. John Wiley and Sons, Chichester, UK.

22.Williams, S.C., Y. Hong, D.C.A. Danavall, J.M.H. Howard, D. Gibson, M.E. Frischer and P.G. Verity. 1998. Distinguishing between living and nonliving bacteria: evaluation of the vital stain propidium iodide and its combined use with molecular probes in aquatic samples. J. Microbiol. Methods 32:225236.

Received 28 March 2000; accepted 5 July 2000.

\author{
Address correspondence to: \\ Dr. John H. Andrews \\ University of Wisconsin \\ Department of Plant Pathology \\ 1630 Linden $\mathrm{Dr}$. \\ Madison, WI 53706, USA \\ e-mail: jha@plantpath.wisc.edu
}

